\title{
On Fast Spectral Solving Triharmonic Equation with Simply Supported Type Condition
}

\author{
Agah D. Garnadi \\ Department of Mathematics, \\ Faculty of Mathematics and Natural Sciences, \\ Bogor Agricultural University \\ Jl. Meranti, Kampus IPB Darmaga, Bogor, 16680 Indonesia
}

\begin{abstract}
This note is addressed to fast solving simply supported boundary value problem of triharmonic equation in the unit rectangle using spectral method.

Key words: Triharmonic equation, simply supported boundary value problem, spectral method.
\end{abstract}

\section{Introduction}

This note is an extension of a work by Fortunato and Townsend [6] on fast solver for Poisson equation to simply supported boundary value problem of Triharmonic equation. Henrici's work [2] on fast solver for Poisson's equation.

The need to solve discretization of Triharmonic equation is motivated by its occurence from computer graphics [7], outside of classical applications such as elasticity and fluid flows $[1,3]$.

2 Triharmonic equations with simply supported boundary conditions.

Suppose that $S$ is a unit square,

$$
S=\{(\xi, \eta) ; 0 \leq \xi, \eta \leq 1\} .
$$


We are going to solve biharmonic equation:

$$
\Delta^{3} u(x, y)=f(x, y)
$$

in the rectangle $S$. Furthermore, we deal with homogeneous simply supported boundary value problem,

$$
\left.\begin{array}{l}
u=0, \\
\Delta u=0, \\
\Delta^{2} u=0,
\end{array}\right\} \text { on } \partial \Omega .
$$

Rewrite as coupled system:

$$
\Delta u(x, y)=v ; \quad \Delta v(x, y)=w(x, y), \quad \Delta w(x, y)=f(x, y),
$$

with the boundary condition:

$$
\left.\begin{array}{l}
u=0, \\
v=0, \\
w=0,
\end{array}\right\} \text { on } \partial \Omega .
$$

To solve the problem using spectral methods on $S$, is easily executed since the availability of fast spectral method for Poisson Equation as given by [6].

\section{Epilogue}

We show an algorithmic development of solving Triharmonic equations in unit rectangle. The implementation of spectral fast solver of Poisson equations in MATLAB in [6], made the fast spectral Triharmonic problem with simply supported conditions is realizable.

\section{References}

[1] M.M. Gupta, Numerical methods for viscous flow problems. In: A.S. Mujumdar(eds.), Advances in Transport Processes, Wiley Eastern, New Delhi, 1980.

[2] P. Henrici, Fast Fourier methods in computational complex analysis, SIAM Rev. 21 (1979) 481-527.

[3] I. Sneddon, Elements in Partial Differential Equations, McGraw-Hill, Tokyo, 1984.

[4] G.D. Smith, Numerical Solution of Partial Differential Equations, Oxford Univ. Press, London, 1975.

[5] Strikwerda, J.C.,2004, Finite difference schemes and partial differential equations, SIAM

[6] Fortunato, Daniel and Townsend, Alex, 2017, Fast Poisson solvers for spectral methods, ,arXiv preprint arXiv:1710.11259 
[7] Alec Jacobson, Elif Tosun, Olga Sorkine and Denis Zorin, 2010, Mixed Finite Elements for Variational Surface Modeling, Eurographics Symposium on Geometry Processing 2010, Volume 29 (2010), Number 5. 